\title{
Reducción de lesiones por caídas relacionadas a reacondicionamiento del hogar
}

Decreased injuries from falls related to home modifications

Keall, M. y col. Lancet 2015;385(9964): 231-38.

\section{Objetivos}

Evaluar la efectividad de las modificaciones en el hogar en la prevención del riesgo de sufrir caídas.

\section{Diseño}

Ensayo clínico aleatorizado por cluster, simple ciego.

\section{Lugar}

Nueva Zelanda (Taranaki).

\section{Pacientes}

Los participantes para ser elegidos debían vivir en una vivienda propia construida antes de 1980 y al menos uno de sus miembros debía recibir un subsidio del estado. Las viviendas fueron aleatorizadas por un método electrónico en 2 grupos: las que recibieron modificaciones en el hogar inmediatas (grupo intervención) y 3 años previos a las modificaciones (grupo control).

\section{Intervención}

Se reclutaron 842 viviendas, 436 fueron aleatorizadas al grupo intervención y 406 al grupo control. Las modificaciones consistían en: pasamanos en escaleras internas y externas, barandas en las escaleras y baños, cerrojos de ventanas, alfombra de baño antideslizante y adecuada iluminación entre otras. Se completó una mediana de 1.148 días de observación (Rango intercuartilo [RIC] 1.085 a 1.263).

\section{Medición de resultados principales}

Se determinó tasa de caídas no intencionales en el hogar por persona por año que requirieron tratamiento médico. Como resultado secundario se midió la tasa de lesiones causadas por caídas en el hogar por año. El análisis se realizó por intención de tratar.

Tabla 1. Riesgo de sufrir lesiones por caídas*

\begin{tabular}{l|c|c|c|c|c|}
\hline & \multicolumn{2}{|c|}{ Grupo intervención } & \multicolumn{2}{|c|}{ Grupo control } \\
\cline { 2 - 5 } & Lesiones $(\mathbf{n})$ & Tasa por persona-año & Lesiones (n) & Tasa por persona-año \\
\hline Lesiones por caídas (totales) & 182 & $0,061(0,006)$ & 192 & $0,072(0,007)$ \\
\hline Lesiones específicas (totales) & 53 & $0,018(0,003)$ & 73 & $0,86(0,66$ a 1,12) \\
\hline
\end{tabular}

*Resultados no ajustados. RR: Riesgo relativo. IC95\%: Intervalo de confianza del 95\%

\section{Resultados principales}

El mayor número de caídas se produjo en las personas $>70$ años. Ver los resultados principales en la tabla 1.

Comparado con el grupo control se estimó una reducción del 26 $\%$ en la tasa de lesiones causadas por caídas en el hogar por año luego de ajustar por edad, caídas previas, sexo y etnia RR 0,74 (IC95\% 0,58 a 0,94). En cuanto a las lesiones totales se estimó una reducción del 39\% por año de exposición RR 0,61 (IC95\% 0,41 a 0,91)

\section{Conclusiones}

Las modificaciones de bajo costo en el hogar reducen lesiones en la población general, Se requieren más investigaciones para identificar cuáles son las modificaciones efectivas del total de las realizadas.

Fuente de financiamiento/conflicto de intereses: Health Research Council of New Zealand/ No presenta.

\section{Comentario}

Las caídas en el hogar son muy frecuentes, especialmente en los adultos mayores. Constituyen una causa importante de internación y de consultas a guardia. El domicilio es el lugar donde estas personas permanecen más tiempo y muchas caídas son evitables a través de la educación del paciente y su familia. La indicación por el profesional de la salud de implementar modificaciones en el hogar muchas veces genera resistencia y dificultad para llevarlas a cabo. Sin embargo pueden resultar efectivas para prevenirlas.
Los resultados de este estudio tienen implicancia para la salud pública, para promover y diseñar programas focalizados en modificaciones ambientales.

\section{Conclusiones del comentador}

Las modificaciones en el hogar reducen las caídas, Poder facilitar a los pacientes estos elementos de adaptación para un hogar más seguro constituyen una estrategia muy valiosa para implementar.

Tami Guenzelovich [Programa sociosanitario del adulto mayor frágil del Hospital Italiano de Buenos Aires. tami,guenzelovich @ hospitalitaliano,org,ar]

Guenzelovich T. Reducción de lesiones por caídas relacionadas a reacondicionamiento del hogar. Evid Act Pract Ambul. 2017;20(1):6. Comentado de: Keall MD, y col. Home modifications to reduce injuries from falls in the home injury prevention intervention (HIPI) study: a cluster-randomised controlled trial. Lancet. 2015;385(9964):231-8. PMID: 25255696.

\section{Referencias}

1. Ministerio de Sanidad, Servicios Sociales e Igualdad Gobierno de España, Boletín informativo: prevención de las caídas en las personas de edad avanzada. 2012. 2. Universidad de Barcelona, Ministerio de trabajo y asuntos sociales Gobierno de España, Adaptación de la vivienda en la población dependiente: necesidades, soluciones y costos. 2007 